\title{
When the light bulb turns on: motivation and collaboration spark the creation of ideas for theoretical physicists
}

\author{
Molly Griston \\ Department of Physics and Astronomy, University of Rochester \\ Jesus Botello \\ Department of Physics, The University of Texas at Austin \\ Michael Verostek \\ Department of Physics and Astronomy, University of Rochester and \\ School of Physics and Astronomy, Rochester Institute of Technology \\ Benjamin M Zwickl \\ School of Physics and Astronomy, Rochester Institute of Technology
}

In this project, we sought to uncover the cognitive processes and skills that are involved in completing a theoretical physics project. Theoretical physics is often portrayed as a field requiring individual genius and can seem inaccessible to undergraduate students, as well as the public. We drew upon the foundations of Cognitive Task Analysis and completed semi-structured interviews with eleven theoretical physics faculty members from several different research institutions who specialized in subfields including quantum optics, biophysics, computational astrophysics, and string theory. We analyzed the processes and skills of these physicists, focusing on an analysis of idea origin, which is typically the first cognitive process within a project, and how it was connected to collaboration and motivation. We used concept maps to organize these codes and portray the factors that influence the creation of project ideas. We found that motivation and collaboration are fundamental determinants of project ideas and their origins, which contradicts the "lone genius" stereotype. These findings on cognitive processes and skills can help us understand how to better prepare students to do theoretical physics research. Finally, the information gathered during this project may be useful for improving the public understanding of theoretical physics, dispelling the belief that the field requires "genius," and making it accessible to more students. (Supported by NSF DGE-1846321 and REU-1757477)

\footnotetext{
2021 PERC Proceedings edited by Bennett, Frank, and Vieyra; Peer-reviewed, doi.org/10.1119/perc.2021.pr.Griston Published by the American Association of Physics Teachers under a Creative Commons Attribution 4.0 license. Further distribution must maintain the cover page and attribution to the article's authors.
} 


\section{INTRODUCTION}

Theoretical physics is often portrayed as a field requiring "genius" and can seem inaccessible to undergraduate students and the public. It is also quite difficult for undergraduate students to get experience in and understand what it means to do theoretical physics research. Previous work has been done to investigate the processes of physicists, but it did not differentiate between experimental and theoretical physicists [1]; it is important to study theoretical physicists in their own right because the majority of undergraduate physics courses are theory-based. This also allows us to study the relationship between theorists and experimentalists. The analysis presented here is part of a larger project performing an in-depth look at the cognitive processes and skills of theoretical physicists. The goal of this study is to identify and understand these processes and skills and determine how we can better prepare undergraduate students to do physics theory. Additionally, we hope to improve public understanding of theoretical physics and make it accessible to more students.

In this specific analysis, we focus on the portion of the cognitive process pertaining to the origination of ideas. Our research is driven by the following questions:

- How do theoretical physicists' ideas originate?

- How does collaboration affect the ways in which project ideas originate?

- How does motivation drive project ideas?

To answer these questions, we used the framework of Cognitive Task Analysis to conduct semi-structured interviews with theoretical physicists. We then went through several rounds of coding and further analysis to better understand the relationship between idea origin, collaboration, and motivation. We hope that the results can provide information on how to better prepare students to do theoretical physics research, and more generally to engage in cognitive processes involved in the exploration of knowledge.

\section{BACKGROUND}

Previous work has included investigations into the research processes of physicists as a whole [1], of students engaging in undergraduate research [2], and of scientists and engineers from several disciplines [3]. However, we focus on the processes of theorists. Combining these prior results with our analysis will provide a fuller picture on how to teach scientific inquiry.

One framework that is often used to analyze cognitive processes is Cognitive Task Analysis (CTA). CTA is a broad category of methods that seek to identify the cognitive skills utilized in task performance. There are many methods of CTA, but they typically share the same general steps. 1. Collect preliminary knowledge: get familiar with the knowledge domain. 2. Identify knowledge representations: choose between flow charts, concept maps, semantic nets, etc. to represent the different sub-tasks and the types of knowledge involved in completing them. 3. Apply focused knowledge elicitation methods: use various techniques to collect the knowledge previously identified. 4. Analyze and verify data collected: data is coded for the purposes of summarizing, categorizing, and synthesizing. 5. Format results for the intended application: results are formatted in a way that they demonstrate the cognitive skills and processes being used [4].

The biggest difference between CTA methods comes during the knowledge elicitation step. The two questions to ask when deciding on a method are "How to look?" and "Where to look?" "How to look?" refers to the different ways of eliciting knowledge, such as interviews, self-reports, observation, and automated capture. The second question, "Where to look?" is further broken down into four categories, most of which are primarily self-explanatory. "Where in time?" refers to the idea that we can ask an individual about an event that has previously happened, one that is currently happening, or one that is taking place in the future. "Where in realism?" refers to the asking about a real world event versus a simulation or scenario that did not actually happen. "Where in difficulty?" refers to asking about routine tasks versus challenging tasks, and "Where in generality?" asks if we want to know about abstract knowledge or specific events. There are positives and negatives to answering each way on all of these questions, so it really comes down to what the data target is and how the information is going to be used $[4,5]$.

CTA can be used with a combination of different methods tailored to research objectives. The methods also vary significantly by the amount of resources, including time required, which will also play a role in choosing the best method. One method that is particularly relevant for our purposes is Applied Cognitive Task Analysis (ACTA), which was designed to elicit process-based information from subject matter experts and takes into account that the interviewer will not have field-specific expertise equivalent to that of the interviewee. $[5,6]$.

\section{METHODS}

While physicists are often divided into theorists and experimentalists, there is no strict dividing line, especially considering fields like computational physics. For our purposes, we decided it was sufficient to rely on each subject's selfidentification as a theoretical physicist. While eleven interviews were conducted, this analysis focuses on five faculty members from three research institutions who do research in the following fields: quantum optics, biophysics, computational astrophysics, and string theory. Three subjects identified as female and two as male, and two identified as Asian, two as White, and one as Latinx. Four of the subjects were associate professors and one was a full professor. We interviewed faculty members of different backgrounds to get a variety of perspectives, but we recognize that the lack of diversity among career stages may limit the generalizability of our results. 
The interview protocol was based on Applied Cognitive Task Analysis. The interview consisted of three sections: the Task Diagram, Knowledge Elicitation, and General Questions. In the Task Diagram section, we asked the subject to recall a recently completed research project and describe the main stages of the process, from start to finish. In the Knowledge Elicitation section, we asked a series of more in-depth questions designed to capture the cognitive processes used by the physicists at various stages of this project. Finally, we asked them more general questions about their career and research life. In all, the interviews lasted approximately 60 to 90 minutes. The data analysis presented here is substantially based on data collected in the Knowledge Elicitation section, specifically answers to these interview questions:

- Where did the research goal originate?

- Was this a standard way for a project to originate?

- How did you know this project was doable and significant?

We also referenced the description of the idea origin process as given in the Task Diagram.

Once the interviews and transcripts were completed, we started the coding and analysis process. The first stage was to use a priori codes for idea origin, motivation, and collaboration, which align with our research questions. We largely ignored other portions of the data, such as the specifics on mathematics usage. Next, we performed Initial Coding on these excerpts [7]. Then, we did second round Focused Coding, which is essentially categorization, on idea origin, motivation, and collaboration separately [8]. Finally, we created concept maps, using the second round codes as nodes, in order to connect the codes and portray relationships. We started with one overarching concept map, which centered around idea origin but also included the related codes from motivation and collaboration. We then made smaller concept maps to address each of the major themes in a more understandable format.

\section{RESULTS}

\section{A. Idea Origin}

The concept map in Fig. 1 shows the fundamental factors that affect project ideas; the nodes are second round categories based on the coded excerpts. This concept map is intended to answer the first research question, "How do theoretical physicists' ideas originate?" As can be seen in Fig. 1 , ideas are generated through a variety of methods, from inspiration from previous work to the identification of an engineering problem to solve. The idea origin process is not limited to simply coming up with a new project idea but is also affected by experimental constraints and general solvability. Finally, project ideas originate from collaborations and are determined by the motivations of the physicist. Figure 1 is significantly simplified, and these connections become more apparent when looking at the more detailed maps, such as in Fig. 2. Many factors that are important to the origin of project ideas are not often found in a traditional undergraduate physics curriculum (e.g., determining if a problem is solvable), which is something noteworthy in all the concept maps.

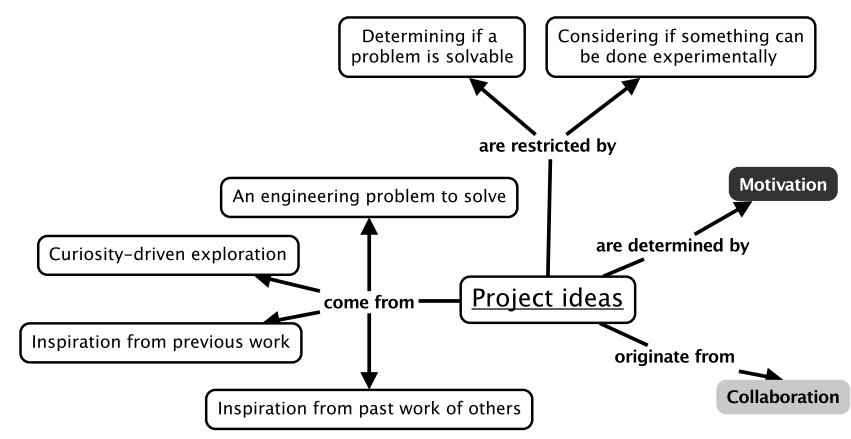

FIG. 1. Concept map centered around idea origin in which the nodes are second round categories

To get a better understanding of what these codes actually mean and how they relate to idea origin, it is worth looking at a paraphrased account of how the project idea originated for one of our subjects. Initially, he was simply intrigued by a certain nonlinear equation from a previous paper of his. He spent a while "playing around" with the equation, trying different methods of "getting at it," but after getting nowhere, he put it on the back burner. Soon after, he spoke to a collaborator about a completely different project and ended up doing some background research related to the work the collaborator was doing. While doing background research in a textbook, he stumbled upon the same equation he had been so intrigued by weeks earlier; however, it was in the context of a completely different system. This mathematical parallel led him to recognize the connection between systems and to quickly understand the physical implications of this connection. This parallel between systems was the basis for his project. In this example, we can see "inspiration from previous work," as his origin point was a previous paper of his, "curiosity-driven exploration" of the nonlinear equation, and "collaboration." Specifically, he partakes in "recognizing connections between systems," which is a node found in Fig. 2.

\section{B. Collaboration}

In Fig. 2, we can see a more detailed concept map, which is designed to show the relationship between collaboration and idea origins and answer the research question, "How does collaboration affect the ways in which project ideas originate?" As stated before, project ideas originate from collaboration, which can occur both directly and indirectly. For example, collaboration can impact idea origin directly when one collaborates with experimentalists who need someone to work on the physics theory corresponding to their experi- 


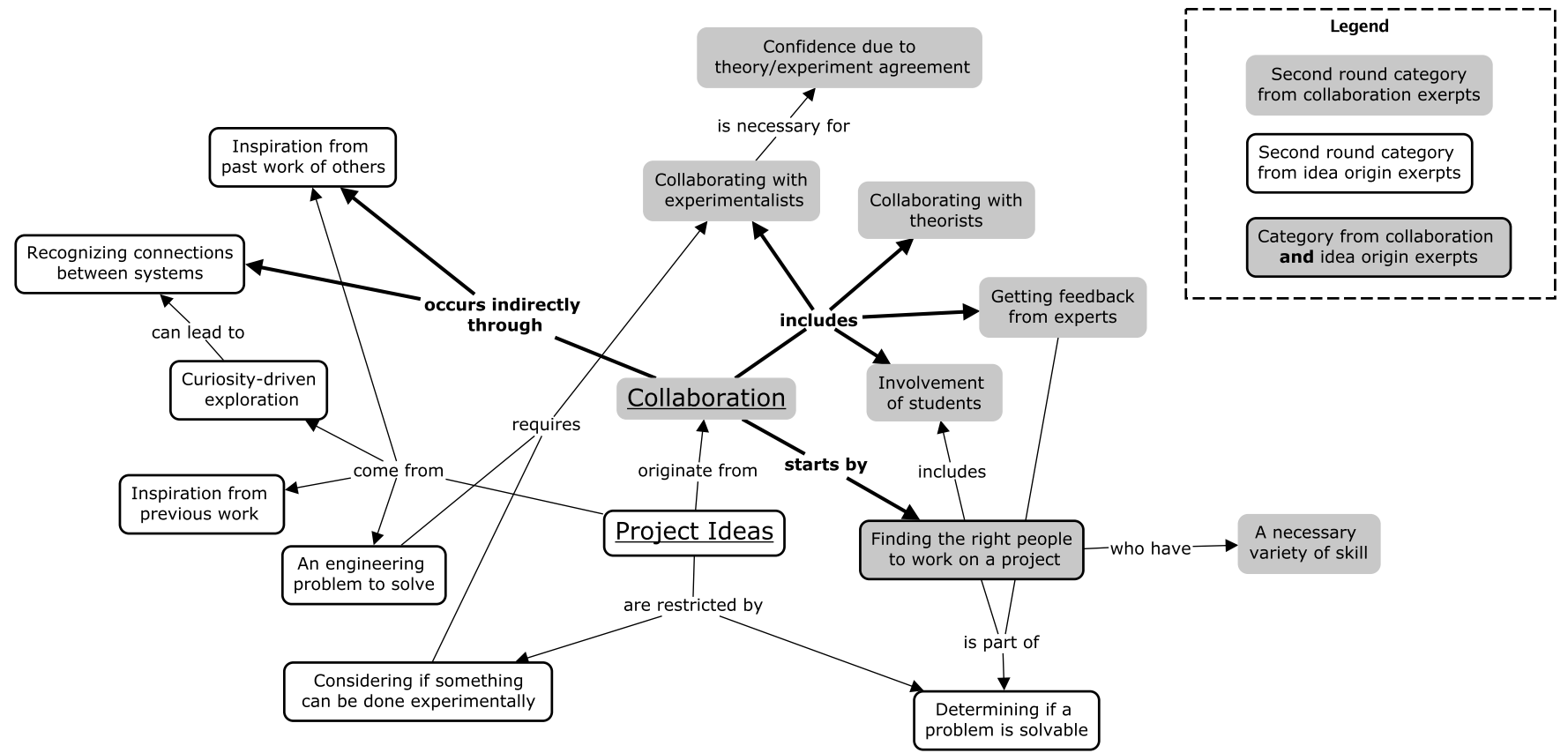

FIG. 2. Concept map which shows the direct and indirect effects that collaboration has on the origination of project ideas. Each node represents a second round code, or category, and is color coded based on the initial code it belongs to. For example, some of the excerpts that were initially coded as 'Collaboration' were then categorized as 'Collaborating with theorists' and others were categorized as 'Involvement of students.'

ment. Other forms of more direct collaboration include collaborating with other theorists, getting feedback from experts, and involving students on a project. However, collaboration can also be more indirect, in the sense that the idea formation comes from other people but not through personal interaction. For instance, someone can get inspiration from the past work of others or recognize a connection between a system they have experience with and one that someone else is working on. While some collaboration is in the form of "I worked directly with another researcher, and we co-authored a paper at the end," often it can look like reading a paper that someone else has written, having an idea of how to add-on to it, and getting feedback from other experts to determine if the problem is doable.

The idea origin process is not limited to simply having the idea, but it also includes determining if the project is doable and worth pursuing. This is another area in which collaboration ties in closely. If someone is working with collaborators, they must assess the abilities of the collaborators (e.g., graduate students or postdocs) to determine if a project is doable for the group. Furthermore, someone may find the need to introduce new collaborators after assessing that the project may not be doable with only their individual skill set.

As stated by one subject, "We don't have ideas in the vacuum." This is certainly represented on the concept map, where almost all of the idea origin nodes quickly connect to a collaboration node. The only one that does not is "inspiration from one's own previous work." However, that previous work had to have an origin, so one can imagine it having an identical concept map of its own. Every physicist that we have interviewed has discussed collaboration being part of their idea origin process.

\section{Motivation}

The analysis intended to answer the research question, "How does motivation drive project ideas?" was also organized into a concept map, although it is not shown here. Faculty typically had extrinsic motivation, such as getting published, as well as more intrinsic motivations such as understanding the physical world or enjoyment of solving problems. One subject summarized some of their motivators as, "You also don't want to pick a problem which is insoluble, so you can break your head on it all your life, but there won't be any papers. Your students won't be able to graduate, etc, etc." This is an example of a faculty member motivated by publishing papers in order to support her students' career success. Another motivation for some theorists, especially those who work more closely with experimentalists, was connecting the theory to something "real" and producing an outcome that is beneficial to people. Clearly, these motivations play a part in determining a faculty member's project ideas. 


\section{Relationship between Idea Origin, Collaboration, and Motivation}

Finally, we examine a case study that exemplifies the interrelationship of idea origin, motivation, and collaboration. The subject in this case study is a theoretical physicist doing quantum optics research.

Idea Origin: In the subject's own words, he first became involved in the project when "[a faculty member] and his then $\mathrm{PhD}$ student...showed up at [his] office one day and asked [him] if [he] knew how to model these things quantum optically." He insists that he didn't think up the project, rather "there was an engineering problem, or an engineering design opportunity, that came [his] way." Referring to Fig. 1, the project idea came from "an engineering project to solve."

Collaboration: The subject collaborated with the aforementioned experimental physics faculty member and his $\mathrm{PhD}$ student; additionally, he worked at a national research lab for the majority of this project. Referring to Fig. 2, the subject's collaboration included extensive "collaborating with experimentalists" and the "involvement of students." His connection to the project was also an effect of "finding the right people to work on a project."

Motivation: The subject discussed a variety of motivators, but the most prevalent one was having a "worthwhile practical output." More generally, he said that he "seek[s] out problems that are connected to some kind of engineering project because [he] think[s] that that's the most impactful kind of work that [he] can do with [his] background." The applied research focus of the national lab also brought together this motivational factor of solving "practical" problems and provided an environment for collaboration with experimentalists and engineers.

Looking at these three pieces together, we can see a coherence between his collaboration with experimentalists, who were the ones who proposed the engineering problem, and their shared motivation to connect theory to something "real." The project idea is directly tied to the collaborations and motivation of the subject, and the three are intrinsically linked. When we ask the question, "How do theoretical physicists' ideas originate?" we cannot answer it without talking about motivation and collaboration.

\section{CONCLUSION}

While theoretical physics is often stereotyped as a "lone genius" field, our data supports the conclusion that collaboration and motivation are fundamental determinants of project ideas and their origins. Specifically, ideas originate through a combination of inspiration from one's previous work and previous work of others, curiosity driven exploration, engineering problems to solve, and more. These origins are all directly tied into what motivates the physicist and are both causes and effects of their collaborations. While a thorough comparison of the processes of theoretical physicists, experimental physicists, and physicists in general is beyond the scope of this paper, we do see overlap between our results and the previously identified research motivations of physicists [1]. In addition, our analysis allows us to gain additional insight into the role of collaboration, particularly between theorists and experimentalists.

These results suggest several ideas about how to better prepare students to do theoretical physics research. First, the opportunity to generate ideas needs to be part of students' education experiences. This structure can be implemented in a level appropriate for students by focusing on the prevalent themes in our data. For instance, a curriculum can be put in place that encourages students to draw inspiration from their own previous work, explore topics they are curious about, and find applications that are important to them. In connection to lab environments, these tasks are similar to the cognitive tasks identified as being part of the experimental research process but often absent from instructional laboratory courses [9]. Second, theoretical projects should include collaborative elements. For example, students may become "experts" on different portions of a topic, including both experimental and theoretical portions, and then several students come together to collaborate and get a fuller understanding of the topic in question. Then, they can use the more comprehensive knowledge to generate potential ideas for how they can advance their cumulative understanding of the topic. Alternately, students can become "experts" on different related systems (e.g., damped mass-spring system or an RLC circuit) and collaborate to see how learning about a different system can help them make better sense of the other one (e.g., a Fabry-Perot resonator in optics). Third, it is important to consider students' motivation when creating theoretical assignments. Faculty benefit from high intrinsic motivation. Students who are motivated by a desire to learn more about a topic or solve a problem may have very different project ideas and processes than those who are only motivated by grades. It may also be helpful to encourage students to be aware of their own motivations, provide them with learning opportunities to act on those motivations, and reflect on how motivation affects their process. Overall, this study has provided insight into the idea origin process of theoretical physicists and allows reflection on how we can prepare students to do physics theory and "think like theoretical physicists."

\section{ACKNOWLEDGMENTS}

This work is supported by NSF Award DGE-1846321 and REU-1757477. 
[1] J. Park, K.-A. Jang, and I. Kim, An analysis of the actual processes of physicists' research and the implications for teaching scientific inquiry in school, Research in Science Education 39, 111 (2009).

[2] N. G. Holmes and C. E. Wieman, Examining and contrasting the cognitive activities engaged in undergraduate research experiences and lab courses, Phys. Rev. Phys. Educ. Res. 12, 020103 (2016).

[3] A. Price, C. Kim, E. Burkholder, A. Fritz, and C. Wieman, A detailed characterization of the expert problem-solving process in science and engineering; guidance for teaching and assessment (2021), arXiv:2005.11463 [physics.ed-ph].

[4] R. Clark, D. Feldon, J. J. G. Van Merrienboer, K. Yates, and S. Early, Cognitive task analysis, Handbook of Research on Educational Communications and Technology , 577 (2008).
[5] B. Crandall, G. A. Klein, and R. R. Hoffman, Working Minds: A Practitioner's Guide to Cognitive Task Analysis (MIT Press, Cambridge, UNITED STATES, 2006).

[6] L. G. Militello and R. J. B. Hutton, Applied cognitive task analysis (ACTA): a practitioner's toolkit for understanding cognitive task demands, Ergonomics 41, 1618 (1998).

[7] J. Salda $\tilde{n}$ a, First cycle coding methods, in Coding Manual for Qualitative Researchers (SAGE Publications Ltd) 2nd ed.

[8] J. Salda $\tilde{n}$ a, Second cycle coding methods, in Coding Manual for Qualitative Researchers (SAGE Publications Ltd) 2nd ed.

[9] C. Wieman, Comparative cognitive task analyses of experimental science and instructional laboratory courses, The Physics Teacher 53, 349 (2015), https://doi.org/10.1119/1.4928349. 